УДК 517.55

\title{
On the Cauchy Problem for Multidimensional Difference Equations in Rational Cone
}

\author{
Tatiana I. Nekrasova* \\ Institute of Mathematics and Computer Science \\ Siberian Federal University \\ Svobodny, 79, Krasnoyarsk, 660041
}

Russia

Received 10.02.2015, received in revised form 15.03.2015, accepted 28.04.2015

The Cauchy problem for multidimensional difference equations in rational cone is formulated and sufficient condition for its solvability is given. The notion of multisection of multiple Laurent series with the support in a rational cone is defined. The formulae which express any multisection through original series are presented.

Keywords: Cauchy problem, rational cone, generating function, multisection.

\section{Introduction}

In this paper we discuss some issues related to the Cauchy problem for multidimensional difference equations whose solutions are sought at the intersection of rational cone $K$ with integer lattice. Methods of the theory of generating functions (z-transformations) play an important role in the study of the Cauchy problem. Problems of solvability of the Cauchy problem in the positive octant of the integer lattice and the algebraic nature of the generating function of its solution are studied in [1]. When passing from positive octant to more general case of a rational cone difficulties arise. They are associated with the fact that the cone $K$, in general, not unimodular.

In the first section we formulate the Cauchy problem and provide the sufficient condition for its solvability (see Theorem 1). The multi-dimensional analogue of the notion of the multisection of a power series helps us to overcome mentioned above difficulties in study of generating functions (series) with supports in rational cones. This multi-dimensional analogue is defined in the second part of the paper. Relation that represents the multisections of the series in terms of the original series (see Theorem 2) is also presented in the second part of the paper.

\section{On solvability of the Cauchy problem}

Let us introduce complex-valued functions $f(x)=f\left(x_{1}, \ldots, x_{n}\right)$ of integer variables $x_{1}, \ldots, x_{n}$. We define the shift operators $\delta_{j}$ with respect to the variables $x_{j}: \delta_{j} f(x)=$ $f\left(x_{1}, \ldots, x_{j-1}, x_{j}+1, x_{j+1}, \ldots, x_{n}\right)$ and polynomial difference operator of the form

$$
P(\delta)=\sum_{\omega \in \Omega} c_{\omega} \delta^{\omega},
$$

*t.neckrasova@gmail.com

(C) Siberian Federal University. All rights reserved 
where $\Omega \subset \mathbb{Z}^{n}$ is a finite set of points of $n$-dimensional integer lattice $\mathbb{Z}^{n}, \delta^{\omega}=\delta_{1}^{\omega_{1}} \cdots \cdots \delta_{n}^{\omega_{n}}$ and $c_{\omega}$ are constant coefficients of the difference operator.

Let us consider the difference equation of the form

$$
P(\delta) f(x)=g(x), x \in X,
$$

where $f(x)$ is unknown function and $g(x)$ is a function defined on some fixed set $X \subset \mathbb{Z}^{n}$. Subset $X_{0} \subset X$ is called the initial (boundary) set. Let us formulate the problem.

Find function $f(x)$ that satisfies equation (1) and the following equation

$$
f(x)=\varphi(x), x \in X_{0}
$$

for a given function $\varphi(x)$.

It is naturally to designate this problem as Cauchy problem for equation (1) and function $\varphi(x)$ in condition (2) is designated as initial data of the Cauchy problem. The existence and uniqueness of solutions of the Cauchy problem depends on all objects involved in the setting this problem: the difference operator $P(\operatorname{delta})$, set $X$ on which we define function $g(x)$ in equation (1) and set $X_{0}$ on which we define the initial data $\varphi(x)$.

We are interested in problems of the form (1)-(2) that arise in combinatorial analysis. Difference operator in the one-dimensional case (see $[2,3]$ ) is given by $P(\delta)=\sum_{\omega=0}^{m} c_{\omega} \delta_{\omega}, c_{m} \neq 0$, the set $X$ is the set of non-negative integers $\mathbb{Z}_{+}$and the set $X_{0}=\{0,1, \ldots, m-1\}$. Under these conditions problem (1)-(2) obviously has the unique solution. In multidimensional case we usually have $X=\mathbb{Z}_{+}^{n}$ and the choice of set $X_{0}$ depends on the properties of the set $\Omega$ on which the characteristic polynomial $P$ is defined. The problem of correct formulation (formulation that ensures the existence and uniqueness of the solution) of Cauchy problem in the positive octant $\mathbb{Z}_{+}^{n}$ of the integer lattice for difference equation (1) was studied [1]. In addition, there was studied the algebraic nature of the generating function of solution of the difference equation.

In this paper we study the problem of solvability of Cauchy problem (1)-(2), that is, the problem of existence and uniqueness of the function $f(x)$ that satisfies (1)-(2). The function is defined at integer points $K \cap \mathbb{Z}^{n}$ of rational cone $K$. Let us give some needed notations and definitions.

Let us assume that $a^{1}, \ldots, a^{n}$ are linearly independent vectors with integer coordinates $a^{j}=$ $\left(a_{1}^{j}, \ldots, a_{n}^{j}\right), a_{i}^{j} \in \mathbb{Z}$. Rational cone generated by the vectors $a^{1}, \ldots, a^{n}$ is the set $K=\left\{x \in \mathbb{R}^{n}\right.$ : $\left.x=\lambda_{1} a^{1}+\cdots+\lambda_{n} a^{n}, \lambda_{j} \in \mathbb{R}_{+}, j=1, \ldots, n\right\}$. Let us note that this cone is simplicial cone, that is, each element of the cone is uniquely expressed in terms of generators. In addition, simplicial cone is also salient cone, that is, this cone contains no lines. Let us introduce matrix $A$. The columns of this matrix are composed of the vectors $a^{j}$ and $\Delta=\operatorname{det} A$. If $\Delta=1$ then the cone $K$ is a unimodular cone.

Let us define a partial order $\underset{K}{\geqslant}$ between points $u, v \in \mathbb{R}^{n}$ as follows

$$
u \geqslant v \Leftrightarrow u \in v+K
$$

where $v+K$ is the shift of the cone $K$ by the vector $v$. In addition, we write $u \underset{K}{\ngtr} v$ if $u \in K \backslash\{v+K\}$.

Let us fix $m \in \Omega$ and specify problem (1)-(2) as follows: we take the intersection $K \cap \mathbb{Z}^{n}$

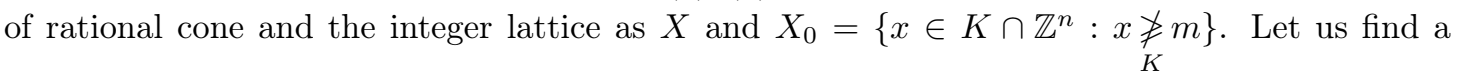
function $f(x)$ that satisfies the equation

$$
P(\delta) f(x)=g(x), x \in K \cap \mathbb{Z}^{n}
$$


and coincides with the given function $\varphi(x)$ on set $X_{0}$ :

$$
f(x)=\varphi(x), x \in X_{0} .
$$

In the positive octant $\mathbb{Z}_{+}^{n}$ of integer lattice (that is $A^{j}=e^{j}, e^{j}$ - unit vectors, $j=1, \ldots, n$ ) degrees of monomials $z^{x}$ on the set of variables are defined as follows $x_{1}+x_{2}+\operatorname{dots}+x_{n}$ and degrees of the monomials are the same when their exponents lie on the hyperplane $x_{1}+x_{2}+$ $\cdots+x_{n}=d$. Note that $\nu=e^{1}+e^{2}+\cdots+e^{n}$ is normal vector to this hyperplane. In the case of an arbitrary simplicial rational cone generated by the vectors $a^{1}, a^{2}, \ldots, a^{n}$ it is natural to take $\nu=a^{1}+a^{2}+\cdots+a^{n}$ and denote $\langle\nu, x\rangle=\nu_{1} x_{1}+\cdots+\nu_{n} x_{n}$.

Theorem 1. If for any $\omega \in \Omega$ the condition $\langle\nu, \omega-m\rangle \leqslant 0$ is fullfield and $m$ is the only point of $\Omega$, which lies on the hyperplane $\langle\nu, x m\rangle=0$, then problem (3)-(4) has the unique solution.

The proof of theorem 1 is reduced to the solvability of an infinite system of linear equations with an infinite number of variables. The system has a feature: each equation contains only a finite number of unknowns. Such system is consistent, if any system of a finite number of equations is consistent (see, [4], Ch. 6, Lemma 6.3.7). We construct sequence of subsystems (3)-(4) that consists of a finite number of equations. These subsystems are arranged so that each following subsystem includes all equations of the previous one. Because of the mentioned above lemma the compatibility of each of these subsystems consistency means that system (3)-(4) is consistent.

Let us introduce the relation $\underset{K}{\prec}$ on the lattice points of rational cone $K$. If $\prec$ is the relation of lexicographical order in $\mathbb{Z}_{+}^{n}$ then for $x, y \in K \cap \mathbb{Z}^{n}$ we define the ratio $\underset{K}{\prec}$ as follows

$$
\underset{K}{\prec} y \Leftrightarrow \Delta A^{-1} x \prec \Delta A^{-1} y
$$

where $A^{-1}$ is the matrix inverse to $A$ and $\Delta=\operatorname{det} A$.

For the vector $\nu$ we consider the linear in $x$ function $\langle\nu, x\rangle, x \in K$. We form the set of its values on the points of the set $K \cap \mathbb{Z}^{n}$ in orderly pattern and designate it as $S$. Note that $S \subset \mathbb{Z}_{+}$ because $\nu$ is in the dual cone to cone $K$. We defined weighted lexicographic ordering $\triangleleft$ on the set of lattice points of the cone $K$ as follows. For $x, y \in K \cap \mathbb{Z}^{n}$ the ratio $x \triangleleft y$ means that $\langle\nu, x\rangle\langle\langle\nu, y\rangle$ and if $\langle\nu, x\rangle=\langle\nu, y\rangle$ then $x \underset{K}{\prec} y$.

Let us take some $s \in S$. Unknown elements of the set are numbered by $J_{s}=\left\{y \in K \cap \mathbb{Z}^{n}\right.$ : $\langle\nu, y\rangle \leqslant s\}$ and equations are numbered by elements of two sets $I_{s}=\left\{x \in K \cap \mathbb{Z}^{n}:\langle\nu, x\rangle \leqslant\right.$ $s-\langle\nu, m\rangle\}$ and $I_{m, s}=\left\{\mu \in X_{0}:\langle\nu, \mu\rangle \leqslant s\right\}$. Points $x$ of the set $I_{s}$ «» are assigned numbers of points $m+x$ in $J_{s}$. Points $x$ of the set $I_{s}$ are numbered in the same way as points $m+x \in J_{s}$. We denote number of elements of a finite set $M$ by $\# M$ then it is easy to see that $\# I_{s}+\# I_{m, s}=\# J_{s}$. Thus, we obtain a system of linear equations for the unknown $f(y), y \in J_{s}$ of the form

$$
\begin{gathered}
\sum_{\omega \in \Omega} c_{\omega} f(x+\omega)=g(x), x \in I_{s}, \\
f(\mu)=\varphi(\mu), \mu \in I_{m, s} .
\end{gathered}
$$

Determinant of system (5)-(6) is denoted by $\Delta m, s$.

Proof of Theorem 1.

All elements in the rows of the determinant $\Delta_{m, s}$ but one are equal to zero. This element is equal to one and it lies on the main diagonal. This follows from the algorithm of ordering of 
unknowns and equations of system (5)-(6). Consider the rows of the determinant corresponding to equations (5). Firstly, only $c_{\omega}$ are equal to zero. Secondly, it follows from the conditions of Theorem 1 that $\omega \triangleleft m, \omega \in \Omega, \omega \neq m$ then $x+\omega \triangleleft x+m$. So in the rows of the determinant that corresponds to equation (5), the last non-zero element is $c_{m}$. Element $c_{m}$ stands on the main diagonal because of the equation has the number $x+m$ and number of unknown is $y=x+m$. Thus, $\Delta_{m, s}$ is the determinant of a lower triangular matrix. Non-zero elements $c_{m}$ are on the main diagonal of the matrix, that is, $\Delta_{m, s} \neq 0$ for all $s \in S$.

Note that in the case $K=\mathbb{R}_{+}^{n}$ theorem 1 is proved with the use of other method (see [1]).

\section{Multisection of Laurent series with the support in a rational cone}

We recall the notion of multisection of power series in the one-dimensional case. For a fixed positive integer $q$ we define the $k$-th $q$-section $T_{k, q}$ of series $G(\xi)=\sum_{j=0}^{\infty} g(j) \xi^{j}$ as follows

$$
T_{k, q} G(\xi)=\sum_{j=0}^{\infty} g(k+j q) \xi^{k+j q}, k=0,1, \ldots, q-1,
$$

where $T_{k, q}$ is a linear operator acting on the ring of formal power series $\mathbb{C}[[\xi]]$. It is known that every $k$-th $q$-section of series is expressed through the original series (see [5]) as follows

$$
T_{k, q} G(\xi)=\frac{1}{q} \sum_{j=1}^{q} r^{q-k j} G\left(r^{j} \xi\right), k=0,1, \ldots, q-1,
$$

where $r$ is a primitive $q$-th root of unity, that is $r^{q}=1, r \neq 1$.

Let us note that multisection is used to prove identities with binomial coefficients and the Bernoulli numbers [5]. The need for a multi-dimensional analogue of the notion of multisection multiple series arises in the study of the Cauchy problem for multidimensional difference equations (see [1,6-9]). In particular, this is the case when supports of generating functions of equation solutions is in rational cone. Generating functions are naturally divided into the sum of multisections. The question arises as to whether the original series and multisections are of the same class, for example, the class of rational or algebraic functions. Let us introduce some needed notations and definitions.

Let $\Lambda=\left\{x \in \mathbb{Z}^{n}: x=\lambda_{1} a^{1}+\ldots+\lambda_{n} a^{n}, \lambda_{i} \in \mathbb{Z}, i=1, \ldots, n\right\}$ be sublattice of $\mathbb{Z}^{n}$ generated by the vectors $a^{1}, \ldots, a^{n}$. We fix $\tau \in \Lambda \cap K$ and introduce $\mu=\left(\mu_{1}, \ldots, \mu_{n}\right)$, where $\mu_{i}$ are coordinates of $\tau$ in the basis $a^{1}, \ldots, a^{n}$. We denote by $\Pi_{\tau}$ the parallelotope $\Pi_{\tau}=\left\{x \in \mathbb{R}^{n}: \underset{K}{\leqslant} x \underset{K}{<}\right\}$ and denote by $\Lambda_{\tau}=\left\{x \in \mathbb{Z}^{n}: x=\lambda_{1} \mu_{1} a^{1}+\ldots+\lambda_{n} \mu_{n} a^{n}, \lambda_{i} \in \mathbb{Z}, i=1, \ldots, n\right\}$ the sublattice of $\mathbb{Z}^{n}$ generated by the vectors $\mu_{1} a^{1}, \ldots, \mu_{1} a^{n}$. Next, we assume that $v$ are points with integer coordinates that belong to parallelotope $\Pi_{\tau}$. The number of points is equal to the volume of parallelotope $\operatorname{Vol}\left(\Pi_{\tau}\right)=\mu_{1} \ldots \mu_{n} \Delta^{n}$. It is obviously that $\bigcup_{v \in \Pi_{\tau} \cap \mathbb{Z}^{n}}\left(v+\Lambda_{\tau}\right)=\mathbb{Z}^{n}$.

Let $\mathbb{C}_{K}[[z]]$ be the ring of Laurent series of the form $F(z)=\sum_{x \in K \cap \mathbb{Z}^{n}} f(x) z^{x}$. Then, the $v$-th $\tau$-section of multiple Laurent series $F(z)$ is a series of the form

$$
T_{v, \tau} F(z)=\sum_{x \in K \cap\left\{v+\Lambda_{\tau}\right\}} f(x) z^{x} .
$$


It is easy to see that any series $F(z)$ from the ring $\mathbb{C}_{K}[[z]]$ can be uniquely expressed as the sum

$$
F(z)=\sum_{v \in \Pi_{\tau} \cap \mathbb{Z}^{n}} T_{v, \tau} F(z)
$$

The following theorem generalizes relation (7) to the case of multiple series.

Theorem 2. Every $v$-th $\tau$-section $T_{v, \tau} F(z)$ is expressed in terms of the original series $F(z)$ as follows

$$
T_{v, \tau} F(z)=\frac{1}{\mu_{1} \ldots \mu_{n} \Delta^{n}} \sum_{J} R^{\tau-v \odot J} F\left(R^{J} z\right),
$$

where $R=\left(R_{1}, \ldots, R_{n}\right), R_{j} \neq 1, j=1, \ldots, n$ is some solution of the system of equations

$$
R^{\mu_{i} a^{i}}=1, i=1, \ldots, n
$$

and $J=\left(j_{1}, \ldots, j_{n}\right), 1 \leqslant j_{1} \leqslant \mu_{1} \Delta, \ldots, 1 \leqslant j_{n} \leqslant \mu_{n} \Delta$, where $\mu_{i}$ are coordinates of $\tau$ in the basis $a^{1}, \ldots, a^{n}$.

From (10) we immediately get

Corollary 1 If the series $F(z)$ is a rational (algebraic) series then the series $T_{v, \tau} F(z)$ for any $\tau \in \Lambda \cap K$ and $v \in \Pi_{\tau}$ is rational (algebraic) series.

Let us consider first the case $K=\mathbb{R}_{+}^{n}$, that is, the case of multiple it power series. Let $\xi=\left(\xi_{1}, \ldots, \xi_{n}\right) \in \mathbb{C}^{n}, \xi^{\lambda}=\xi_{1}^{\lambda_{1}} \ldots \xi_{n}^{\lambda_{n}}$. We fix $q=\left(q_{1}, \ldots, q_{n}\right) \in \mathbb{Z}_{+}^{n}$ and consider the half-open parallelepiped $\Pi_{q}=\left\{x \in \mathbb{R}_{+}^{n}: 0 \leqslant x<q\right\}$. Number of points $k \in \Pi_{q} \cap \mathbb{Z}^{n}$ with integer coordinates is equal to $\# \Pi_{q} \cap \mathbb{Z}^{n}=q_{1} \cdot \ldots \cdot q_{n}$. Integer lattice $\mathbb{Z}^{n}$ can be written as $\mathbb{Z}^{n}=$ $\bigcup_{k \in \Pi_{q} \cap \mathbb{Z}^{n}}\left(k+q \mathbb{Z}^{n}\right)$, where the union is taken over all shifts of sublattice $q \mathbb{Z}^{n}=\left(q_{1} \mathbb{Z}\right) \times \cdots \times\left(q_{n} \mathbb{Z}\right)$ by vectors $k \in \Pi_{q} \cap \mathbb{Z}^{n}$.

Let us denote

$$
\mathcal{F}(\xi)=\sum_{\lambda \in \mathbb{Z}_{+}^{n}} h(\lambda) \xi^{\lambda} \in \mathbb{C}[[\xi]]
$$

Then from (8) we obtain

$$
T_{k, q} \mathcal{F}(\xi)=\sum_{\lambda \in k+q \mathbb{Z}_{+}^{n}} h(\lambda) \xi^{\lambda} \text {. }
$$

Theorem 3. For $k$-th q-section of the power series (12) the following relation

$$
T_{k, q} \mathcal{F}(\xi)=\frac{1}{q_{1} \cdot \ldots \cdot q_{n}} \sum_{J} r^{q-k \odot J} \mathcal{F}\left(r^{J} \xi\right)
$$

is valid, where $J=\left(j_{1}, \ldots, j_{n}\right), 1 \leqslant j_{1} \leqslant q_{1}, \ldots, 1 \leqslant j_{n} \leqslant q_{n}, r=\left(r_{1}, \ldots, r_{n}\right)$ and $r_{j}$ is a primitive $q_{j}$-th root of unity.

Proof. It is easy to verify that the $k$-th $q$-section of the power series (12) can be written as

$$
T_{k_{i}, q_{i}}^{i} \mathcal{F}(\xi)=\sum_{j \in \mathbb{Z}_{+}^{n}} h\left(j_{1}, \ldots, k_{i}+q_{i} j_{i}, \ldots, j_{n}\right) \xi_{1}^{j_{1}} \ldots \xi_{i}^{k_{i}+q_{i} j_{i}} \ldots \xi_{n}^{j_{n}} .
$$

Note that the last equality can be taken as a definition of multisection.

Sequential execution (composition) of $q_{i}$-th and $q_{j}$-th sections is designated as $T_{k_{i}, q_{i}}^{i} \circ T_{k_{j}, q_{j}}^{j}$. It should be noted that operation $\circ$ is commutative and associative. 
For $k=\left(k_{1}, \ldots, k_{n}\right)$ and $q=\left(q_{1}, \ldots, q_{n}\right)$ the $k$-th $q$-section of multiple power series $\mathcal{F}(\xi)$ is the following series

$$
T_{k, q} \mathcal{F}(\xi)=T_{k_{1}, q_{1}}^{1} \circ \cdots \circ T_{k_{n}, q_{n}}^{n} \mathcal{F}(\xi)=\sum_{j \in \mathbb{Z}_{+}^{n}} h(k+q j) \xi^{k+q i} .
$$

Using (14) and $n$ times (7), we obtain

$$
\begin{gathered}
T_{k, q} \mathcal{F}(\xi)=T_{k_{1}, q_{1}}^{1} \circ \cdots \circ T_{k_{n}, q_{n}}^{n} \mathcal{F}(\xi)= \\
=T_{k_{1}, q_{1}}^{1} \circ \cdots \circ T_{k_{n-1}, q_{n-1}}^{n-1} \sum_{j \in \mathbb{Z}_{+}^{n}} h\left(j_{1}, \ldots, j_{n-1}, k_{n}+q_{n} j_{n}\right) \xi_{1}^{j_{1}} \ldots \xi_{n-1}^{j_{n-1}} \xi_{n}^{k_{n}+q_{n} j_{n}}= \\
=T_{k_{1}, q_{1}}^{1} \circ \cdots \circ T_{k_{n-1}, q_{n-1}}^{n-1} \frac{1}{q_{n}} \sum_{j_{n}=0}^{q_{n}} r_{n}^{q_{n}-k_{n} j_{n}} \mathcal{F}\left(\xi_{1}, \ldots, \xi_{n-1}, r^{j_{n}} \xi_{n}\right)= \\
=T_{k_{1}, q_{1}}^{1} \circ \ldots \circ T_{k_{n-2}, q_{n-2}}^{n-2} \frac{1}{q_{n-1}} \sum_{j_{n-1}=0}^{q_{n-1}} r_{n-1}^{q_{n-1}-k_{n-1} j_{n-1}} \times \\
\times \frac{1}{q_{n}} \sum_{j_{n}=0}^{q_{n}} r_{n}^{q_{n}-k_{n} j_{n}} \mathcal{F}\left(\xi_{1}, \ldots, \xi_{n-2}, r^{j_{n-1}} \xi_{n-1}, r^{j_{n}} \xi_{n}\right)= \\
\frac{1}{q_{1}} \sum_{j_{1}=0}^{q_{1}} r_{1}^{q_{1}-k_{1} j_{1}} \ldots \\
\sum_{q_{n-1}} \sum_{j_{n-1}=0}^{q_{n-1}} r_{n-1}^{q_{n-1}-k_{n-1} j_{n-1}} \frac{1}{q_{n}} \sum_{j_{n}=0}^{q_{n}} r_{n}^{q_{n}-k_{n} j_{n}} \mathcal{F}\left(r^{j_{1}} \xi_{1}, \ldots, r^{j_{n-1}} \xi_{n-1}, r^{j_{n}} \xi_{n}\right)= \\
=\frac{1}{q_{1} \cdot \ldots \cdot q_{n}} \sum_{J} r^{q-k \odot J} \mathcal{F}\left(r^{J} \xi\right) .
\end{gathered}
$$

Now we apply (13) for the proof of Theorem 2.

Proof of the Theorem 2. We show that (10) is equivalent to (13) after some transformations. On the left hand side of (10) we introduce the change of variables $z^{A}=\xi^{\Delta}$, where $z^{A}=$ $\left(z_{1}^{a_{1}^{1}} \ldots z_{n}^{a_{n}^{1}}, \ldots, z_{1}^{a_{1}^{n}} \ldots z_{n}^{a_{n}^{n}}\right)$. Let us denote $k=\Delta A^{-1} v, q=\Delta A^{-1} \tau$ and note that $q=\Delta \mu$. After change $x=\frac{A \lambda}{\Delta}$ of summation index $x \in K \cap \mathbb{Z}^{n}$ on $\lambda \in \bigcup_{t \in \Pi_{\Delta} \cap \mathbb{Z}^{n}}\left(t+\Delta \mathbb{Z}_{+}^{n}\right)$, where $\Pi_{\Delta}=\left\{y \in \mathbb{R}^{n}: 0 \leqslant y<(\Delta, \ldots, \Delta)\right\}$, we obtain

$$
\begin{aligned}
T_{v, \tau} F(z)= & \sum_{x \in\left\{v+\Lambda_{\tau}\right\} \cap K} f(x) z^{x}=\sum_{\lambda \in \mathbb{Z}_{+}^{n}} f(v+A \lambda \odot \mu) z^{v+A \lambda \odot \mu}= \\
= & \sum_{\lambda \in \mathbb{Z}_{+}^{n}} f\left(\frac{1}{\Delta} A k+A \lambda \odot \frac{q}{\Delta}\right) z^{\frac{1}{\Delta} A k+A \lambda \odot \frac{q}{\Delta}} .
\end{aligned}
$$

Next, we denote

$$
h(\lambda)= \begin{cases}f\left(\frac{1}{\Delta} A \lambda\right), & \text { if } \lambda \in \bigcup_{t \in \Pi_{\Delta} \cap \mathbb{Z}^{n}}\left(t+\Delta \mathbb{Z}_{+}^{n}\right), \\ 0, & \text { if } \lambda \notin \bigcup_{t \in \Pi_{\Delta} \cap \mathbb{Z}^{n}}\left(t+\Delta \mathbb{Z}_{+}^{n}\right) .\end{cases}
$$

Then relation (15) becomes

$$
\sum_{\lambda \in \mathbb{Z}_{+}^{n}} h(k+q \odot \lambda) \xi^{k+q \odot \lambda}=T_{k, q} \sum_{\lambda \in \mathbb{Z}_{+}^{n}} h(\lambda) \xi^{\lambda}=T_{k, q} \mathcal{F}(\xi) .
$$


Now we do the same change of variables $z^{A}=\xi^{\Delta}$ on the right hand side of (10)

$$
\begin{gathered}
\frac{1}{\mu_{1} \ldots \mu_{n} \Delta^{n}} \sum_{J} R^{\tau-v \odot J} F\left(R^{J} z\right)= \\
=\frac{1}{\mu_{1} \ldots \mu_{n} \Delta^{n}} \sum_{J} R^{\frac{1}{\Delta} A q-\frac{1}{\Delta} A k \odot J} \sum_{\lambda \in \bigcup_{t \in \Pi_{\Delta} \cap \mathbb{Z}^{n}}\left(t+\Delta \mathbb{Z}_{+}^{n}\right)} f\left(\frac{1}{\Delta} A \lambda\right) R^{J \frac{1}{\Delta} A \lambda} z^{\frac{1}{\Delta} A \lambda}= \\
=\frac{1}{\mu_{1} \ldots \mu_{n} \Delta^{n}} \sum_{J} r^{q-k \odot J} \sum_{\lambda \in \bigcup_{t \in \Pi_{\Delta} \cap \mathbb{Z}^{n}}\left(t+\Delta \mathbb{Z}_{+}^{n}\right)} f\left(\frac{1}{\Delta} A \lambda\right) r^{J \lambda} \xi^{\lambda}= \\
=\frac{1}{q_{1} \ldots q_{n}} \sum_{J} r^{q-k \odot J} \sum_{\lambda \in \mathbb{Z}_{+}^{n}} h(\lambda) r^{J \lambda} \xi^{\lambda}=\frac{1}{q_{1} \ldots q_{n}} \sum_{J} r^{q-k \odot J} \mathcal{F}\left(r^{J} \xi\right) .
\end{gathered}
$$

Thus, by theorem 2 we find that the right hand side is equal to the left hand side

$$
T_{k, q} \mathcal{F}(\xi)=\frac{1}{q_{1} \ldots q_{n}} \sum_{J} r^{q-k \odot J} \mathcal{F}\left(r^{J} \xi\right)
$$

After returning to the variable $z$ we find that relation (10) is valid.

\section{References}

[1] M.Bousquet-Mélou, M.Petkovšek, Linear recurrences with constant coefficients: the multivariate case, Discrete Mathematics, 225(2000), 51-75.

[2] R.F.Isaacs, A Finite Difference Function Theory, Univ. Nac. Tucuman. Revista A., 2(1941), $177-201$.

[3] A.O.Gelfond, The calculus of finite differences Moscow, KomKniga, 2006 (in Russian).

[4] L.Hörmander, An introduction to complex analysis in several variables, 3 Ed., North Holland, 1990.

[5] J.Riordan, Combinatorial Identities, John Wiley and Sons, New-York, 1968.

[6] E.K.Leinartas, Multiple Laurent series and fundamental solutions of linear difference equations, Siberian Math. J., 48(2007), no. 2, 335-340.

[7] E.K.Leinartas, The stability of the Cauchy problem for the homogeneous difference operator and the amoeba of characteristic set, Siberian Math. J., 52(2011), no. 5, 1087-1095.

[8] T.I.Nekrasova, Cauchy problem for multidimensional difference equations in lattice cone, Journal of Siberian Federal University. Mathematics \& Physics, 5(2012), no. 4, 576-580 (in Russian).

[9] T.I.Nekrasova, Sufficient conditions of algebraicity of generating functions of the solutions of multidimensional difference equations, Izvestiya Irkutskogo Gosudarstvennogo Universiteta, 6(2013), no. 3, 88-96 (in Russian). 


\title{
О задаче Коши для многомерных разностных уравнений в рациональных конусах
}

\section{Татьяна И. Некрасова}

\begin{abstract}
Сформулирована задача Коши для многомерных разностных уравнений в рачиональных конусах, дано достаточное условие ее разрешимости. Определено понятие мультисекции кратных рядов Лорана с носителями, лежащими в рациональных конусах, и приведена формула, выражающая всякую мультисекцию через исходный ряд.
\end{abstract}

Ключевые слова: задача Коши, рачиональный конус, производящая функция, мультисекция. 